\title{
Printing of amorphous and crystalline materials pre-machined using focussed ion beam patterning
}

\author{
C. L. Sones, K. S. Kaur, M. Feinaeugle, J. Y. Ou, K. A. Sloyan, T. C. May- Smith, R. W. Eason \\ Optoelectronics Research Centre, University of Southampton, Highfield, Southampton, U.K, SO171BJ
}

\section{F. Di Pietrantonio, E. Verona}

Institute of Acoustics and Sensors - CNR 100 00133, Rome, Italy

Laser Induced Forward Transfer (LIFT) is a laser-based direct write technique which has been routinely employed for trials of the deposition of organic and inorganic compounds, polymers and biomaterials on various substrates for the realisation of devices such as OLEDs [1]. This single-shot printing approach, which allows a spatially controlled pixel-by-pixel deposition has been widely trialled for printing as it enables operation in a standard ambient environment, has the ability to print a wide range of materials, allows printing of multilayered stacks composed of dissimilar materials, allows printing on both structured and planar substrates, and beam shaping of the incident laser pulse is also possible to further control the size and shape of the deposited material.

However, there are two fundamental problems in LIFT printing which must be addressed if precise and debris-free printing of high quality deposits is to be achieved. The first concerns the unavoidable partial ablation or complete melting of the printed material (termed the donor) due to the absorption of the laser pulse which is responsible for the forward transfer process. The use of a polymer sacrificial layer [2], sandwiched between the donor and the carrier substrate, and referred to as a dynamic release layer (DRL) that decomposes into gaseous fragments to provide the explosive push required to propel the donor towards the receiver, has been employed to alleviate this problem. However a second problem exists which concerns the unavoidable shearing and tearing of the donor around its perimeter, which presents a fundamental limitation both for debris-free transfer and also for donors that are fragile, brittle, of substantial thickness ( $>$ a few $\mu \mathrm{m}$ ), or composite stacks where edge quality or layer mixing is an issue. A method of pre-patterning is therefore highly desirable to precisely define the spatial extent of donor transfer to achieve much more precise and controlled printing results. Pre-patterning is also essential for applications involving LIFTing of thick films, as the edge shearing problem must inevitably result in debris. Currently such fracture around the donor perimeter sets a limit for the thickness of the donor film that can be printed with acceptable edge quality, with a maximum value of $\sim 1 \mu \mathrm{m}$ being reported to date using a DRL [2]. We present in this contribution our initial results for a modified approach that essentially removes these limitations, and helps in the deposition of debris-free material in intact form with extremely smooth edge quality and well-defined shapes.

The pre-patterning step has been achieved using focussed ion-beam (FIB) machining of the donor. It is not necessary to machine throughout the entire depth of the donor as this would prevent the laser-induced pressure build-up behind the material intended for transfer. For an optimum depth of pre-machining, the donor is then transferred onto the receiver substrate resulting in a uniform and clean deposit. Zinc oxide $(\mathrm{ZnO})$, an environmental-friendly lead-free piezoelectric was chosen as the trial donor material for these FIB pre-patterned LIFT experiments, and we report our first results below. The SEM image in Fig.1a shows a circular ring of $5 \mu \mathrm{m}$ diameter pre-machined via FIB to a depth of $0.8 \mu \mathrm{m}$ into a $1 \mu \mathrm{m}$ thick $\mathrm{ZnO}$ donor. Fig. $1 \mathrm{~b}$ shows a printed disc with a $10 \mu \mathrm{m}$ diameter deposited onto a Si receiver using a single pulse of a Ti:sapphire laser operating at $800 \mathrm{~nm}$, at an incident energy density of $450 \mathrm{~mJ} / \mathrm{cm}^{2}$. For comparison, Fig 1c shows the undesirable and in our experience unavoidable shattering associated with a $\mathrm{ZnO}$ pixel deposited with similar laser intensity, but without the essential pre-machining step.

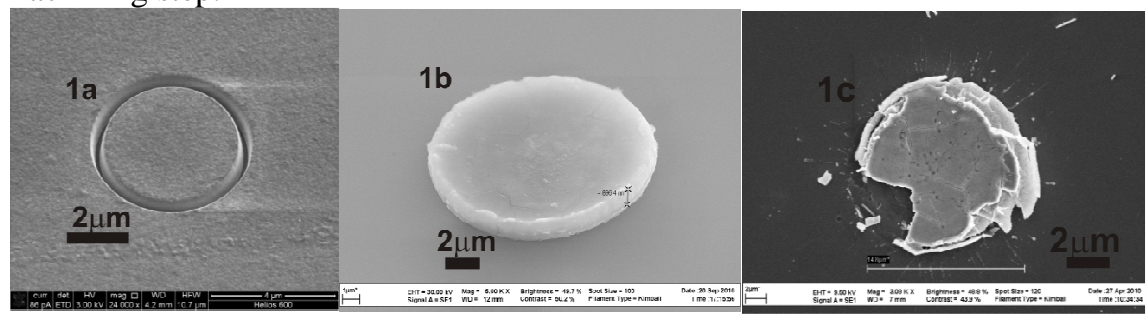

Fig. 1 a) SEM image of a FIB machined ZnO donor; b) deposit LIFTed from this pre-machined donor; c) deposit LIFTed from a non-machined section of the same donor.

The above results show our initial trials and a detailed study to understand the influence of the machining depth on the quality of the deposited pixel is currently underway. We are also investigating the ability of this approach to deposit well-defined pixels from single crystal donor materials and these results will also be presented

\section{References}

[1] J. Bohandy, B.F. Kim, F.J. Adrian, “Metal deposition from a supported metal film using an excimer laser,”J. Appl. Phys. 60, 1538 (1986).

[2] K.S.Kaur, R.Fardel, T.C.May-Smith, M.Nagel, D.P.Banks, C.Grivas, T.Lippert, R.W.Eason, "Shadowgraphic studies of triazene assisted laser-induced forward transfer of ceramic thin films,"J. Appl. Phys., 105, 113119, (2009). 